\title{
Magnetism and Continuum Surveys Working Together
}

\author{
Federica Govoni*, Matteo Murgia, Valentina Vacca, Francesca Loi \\ INAF - ORA Cagliari, Via della Scienza 5, I-09047 Selargius (CA), Italy \\ E-mail: fgovoni@oa-cagliari.inaf.it, matteo@oa-cagliari.inaf.it, \\ vvaccaloa-cagliari.inaf.it, floi@oa-cagliari.inaf.it
}

\section{Luigina Feretti, Gabriele Giovannini, Monica Trasatti}

INAF - ORA Bologna, Via Gobetti 101, I-40129 Bologna, Italy

E-mail: Iferetti@ira.inaf.it, ggiovann@ira.inaf.it, trasattieira.inaf.it

\section{Chiara Ferrari}

Laboratoire Lagrange, UCA, OCA, CNRS, Blvd de l'Observatoire, CS 34229, 06304 Nice cedex

4, France

E-mail: chiara.ferrarieoca.eu

\section{Annalisa Bonafede}

Hamburg Sternwarte, Gojenmbergsweg 112, 21029 Hamburg, Germany

E-mail: annalisa.bonafede@hs.uni-hamburg.de

\begin{abstract}
Magnetic fields are everywhere in space and their presence is believed to play a crucial role in the formation and evolution of many astrophysical objects. Despite of their importance, our knowledge about evolution, structure, and origin of magnetic fields is still poor and numerous questions remain unanswered. The full-Stokes capability of the future high sensitivity and resolution spectro-polarimetric surveys promises a transformational advance in our understanding of cosmic magnetism. These surveys, combined with innovative approaches incorporating observational, theoretical, and numerical techniques, will permit to improve our knowledge of the incidence, strength, and morphology of magnetic fields in the interstellar medium, in the intracluster medium, at the boundary of galaxy clusters, and possibly in the bridges which join clusters in the Cosmic Web. In this review, we will focus on the status of research and future developments related to magnetic fields in galaxy clusters.
\end{abstract}

EXTRA-RADSUR2015 (**)

20-23 October 2015

Bologna, Italy

(**) This conference has been organized with the support of the Ministry of Foreign Affairs and International Cooperation, Directorate General for the Country Promotion (Bilateral Grant Agreement ZA14GR02 - Mapping the Universe on the Pathway to SKA)

*Speaker. 


\section{Introduction}

The presence of magnetic fields in extra-galactic astronomical objects has been unambiguously revealed. They have been detected in spiral galaxies (see e.g., G. Heald; these proceedings), in active galactic nuclei (see e.g., I. Agudo, R. Laing; these proceedings), and in galaxy clusters. In addition, cosmological magneto-hydrodynamical simulations also suggest the possible presence of magnetic fields even on larger scales, in bridges which join galaxy clusters in the Cosmic Web (see e.g., F. Vazza; these proceedings).

In the following, we will show how sensitive radio continuum and polarization observations can work together to improve our knowledge of the incidence, strength, and morphology of magnetic fields in galaxy clusters. Much of what is known about cluster magnetic fields (see e.g., [1, 2, 3] for reviews), comes from observations of extended radio sources diffuse in the intracluster medium (radio halos and relics) and Faraday Rotation Measure (RM) of discrete sources. These observations are usually consistent with central magnetic field strengths of a few $\mu \mathrm{G}$, but stronger fields, with values exceeding $\simeq 10 \mu \mathrm{G}$, are derived in the inner regions of relaxed cooling core clusters. In addition, there are several indications that the magnetic field intensity decreases going from the centre to the periphery following the cluster gas density.

The possibility to obtain RM data in combination with continuum and polarization images of diffuse radio sources in galaxy clusters is fundamental to accurately investigate the magnetic field power spectrum over a large range of spatial scales $[4,5]$. The diffuse radio emission detected in the intracluster medium of galaxy clusters is due to synchrotron radiation, which is a direct probe of relativistic electrons gyrating around magnetic field lines. The observed intensity of the synchrotron emission is related to the field strength, while the fraction of polarized emission is related to the field's degree of ordering. Moreover, Faraday rotation is related to the magnetoionic intracluster medium along the line of sight, so that magnetic fields properties can be derived through the analysis of the RM of radio sources located within or in the background of galaxy clusters. To describe the Faraday rotation, we use the Faraday depth [6] of the intracluster medium:

$$
\phi(\mathbf{l})_{\left[r a d / m^{2}\right]}=812 \int_{0}^{L[k p c]} n_{e\left[c m^{-3}\right]}(l) B_{\|_{[\mu G]}}(l) \mathrm{d} l
$$

where $n_{e}$ is the thermal gas density, $B_{\|}$is the strength of the magnetic field component along the line of sight, and $L$ is the path length along the line of sight. In the simplest possible scenario in which a discrete radio source is located in the background of a galaxy cluster, the RM, which correspond to the Faraday depth calculated along the entire intracluster medium path, is an observational quantity given by the slope of the variation of the polarization angle with $\lambda^{2}$ :

$$
\Psi_{\mathrm{Obs}}(\lambda)=\Psi_{\mathrm{I} n t}+\lambda^{2} \times R M
$$

where $\Psi_{\mathrm{O} b s}(\lambda)$ is the observed polarization angle at a wavelength $\lambda$ and $\Psi_{\mathrm{I} n t}$ is the intrinsic polarization angle. When the intracluster medium emits a polarized radio signal, as in diffuse synchrotron sources in galaxy clusters, the polarized radiation will in general be emitted over a range of physical distances and also over a range of Faraday depths. As a consequence, the polariza- 
tion angle will no longer vary linearly with $\lambda^{2}$ and the emission spectrum in Faraday space can be recovered using the rotation measure synthesis technique (see e.g., $[6,7,8,9,10,11]$ ).

\section{Cluster magnetic fields through the study of discrete sources}

Information on galaxy cluster magnetic fields can be obtained through the analysis of RM images of radio galaxies in the background or in the galaxy clusters themselves, in conjunction with X-ray observations of the hot gas. RM studies were performed in statistical samples (e.g., [12, 13, 14]) as well as in individual galaxy groups (e.g., [15]), merging galaxy clusters (e.g., [4]), and cooling core galaxy clusters (e.g., [16]). These studies reveal that magnetic fields are widespread in the intracluster medium, regardless of the presence of diffuse radio halo emission. In Fig. 1 we present an example of rotation measure image in the cooling core galaxy cluster A2199. As shown in Fig. 1, the RM distribution seen across radio galaxies typically present patchy structures. The observed RM fluctuations indicate that intracluster magnetic fields are not regularly ordered, but turbulent on scales ranging from tens of $\mathrm{kpc}$ to $\lesssim 100 \mathrm{pc}$. Therefore, RM measurements probe the complex topology of cluster magnetic fields. By sampling the rotation measure with radio galaxies located at different projected distances from the cluster center it is possible to investigate the behaviour of the magnetic field fluctuations on large scales (e.g., [17]).

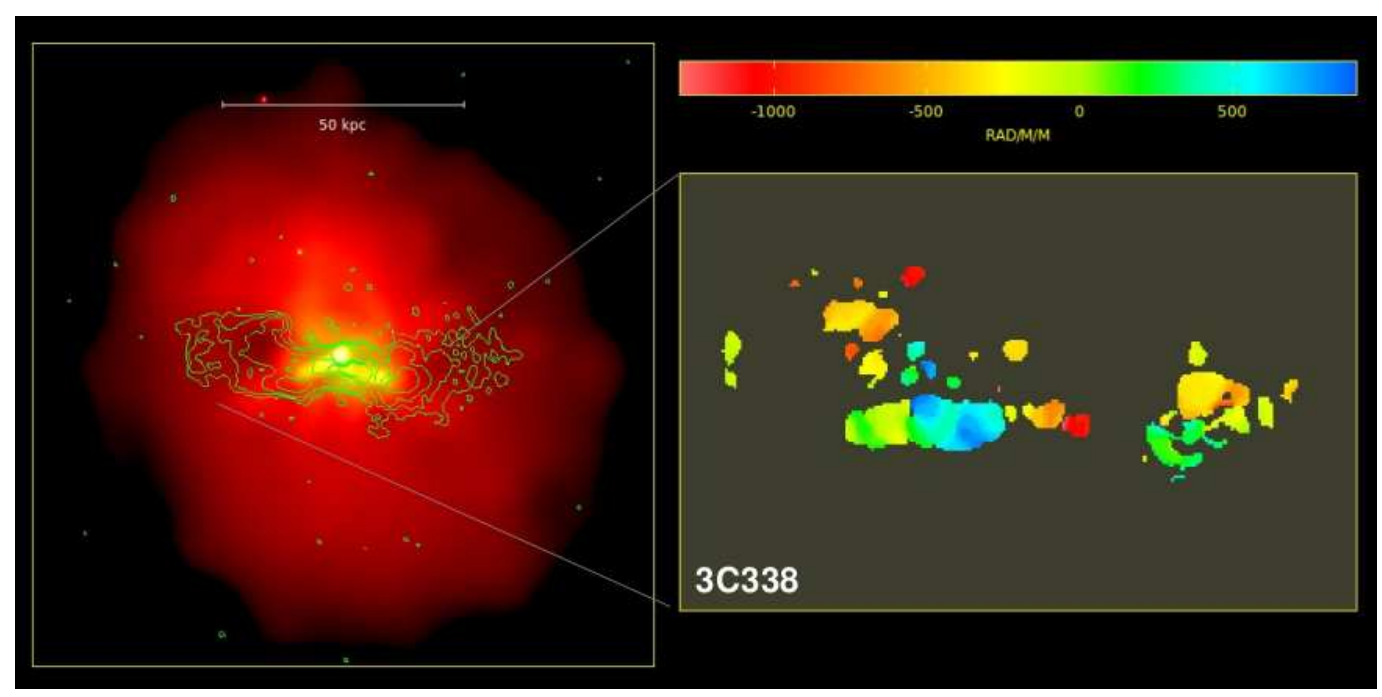

Figure 1: Left panel: Chandra X-ray image of the galaxy cluster A2199 with VLA radio contours of 3C338 overlaid. Pronounced X-ray cavities can be identified at the location of the radio galaxy lobes. Right panel: Rotation measure image of 3C338. Adapted from Vacca et al.(2012) [16] .

State of the art software tools and approaches based on a Fourier domain formulation have been developed to constrain the magnetic field power spectrum parameters on the basis of the rotation measure images [18, 19, 20, 21, 22]. In some galaxy clusters and galaxy groups containing radio sources with very detailed RM images, the magnetic field power spectrum has been estimated. However, the sensitivity of the current facilities limits the RM studies to a few radio galaxies per cluster and few galaxy clusters have been studied in detail up to now $[23,19,24,4,25,20,15,17$, $16]$. 
The densest grid of Faraday rotation measure actually available has been obtained by Taylor et al. (2009) [26]. They analyzed the NRAO VLA Sky Survey (NVSS, [27]) data to derive rotation measures toward 37543 polarized radio sources. The resulting RM catalog contains data on sources distributed roughly equally over the sky at declination larger than $-40^{\circ}$, with an average density of more than one RM per square degree. The RM catalog obtained from the NVSS is not dense enough to derive a detailed description of the strength, structure, and radial decrease of cluster magnetic fields. It is therefore necessary to increase the statistic of RMs toward galaxy clusters.

The goal of conducting a very deep All-sky polarimetric survey on the Square Kilometre Array has been a mainstay for cosmic magnetism science for over a decade [28, 29, 30]. In order to exploit the vast potential for magnetism science, the magnetism community planned a polarisation survey to be performed with SKA1-MID over the entire accessible sky at 950-1760 MHz (Band2) at a resolution of $2^{\prime \prime}$ and down to a sensitivity of $4 \mu \mathrm{Jy} /$ beam [31]. This survey must provide not only continuum and polarisation imaging at high resolution, but also deliver a very dense RM catalog permitting transformational science for almost every class of astronomical object, including galaxy clusters. By extrapolating the current polarization counts $[32,33,34]$ to the sensitivity of the SKA1MID survey, we expect to obtain the RM for 7-14 million discrete extra-galactic sources, i.e. a grid of Faraday RMs $\simeq 200-500 \times$ denser than the RM grid based on the NVSS survey. This will permit to measure the magnetic fields in galaxy clusters, but also in a huge number of intervening galaxies out to high redshifts, to search for a pervasive intergalactic magnetic field, and to investigate the detailed structure of the magnetic field in the Milky Way.

In conclusion, SKA1 would be able to detect RM toward a very large number of radio sources per cluster with a high spatial resolution. To exploit the information from the incoming polarization surveys and RM grids, Bayesian algorithms [35] have been developed capable of separating the extra-galactic contribution intrinsic to the emitting sources from that due to large-scale environments (galaxy clusters, filaments, voids, etc). Applications [36] to mock data indicate that SKA1-MID (Band2) will allow the investigation of extra-galactic magnetic fields associated with the overall large-scale structure at least down to strengths of $\sim 0.1-1 \mathrm{nG} . "$

\section{Cluster magnetic fields through the study of diffuse synchrotron emission}

In recent years, there has been growing evidence for the existence of cluster large-scale diffuse radio sources of synchrotron origin (called halos, mini-halos or relics, depending on their position in the cluster, size, morphology and polarization properties), associated with the intracluster medium (see e.g., A. Bonafede, G. Bernardi, R. Cassano, V. Cuciti, M. Gitti, R. Kale, R. Van Weeren, T. Venturi; this proceedings). Radio halos permeating the center of merging galaxy clusters, provide the most spectacular and direct evidence of the presence of relativistic particles and magnetic fields associated with the intracluster medium. These radio sources, extended over volumes of $\sim 1 \mathrm{Mpc}^{3}$, are diffuse low-surface-brightness and steep-spectrum synchrotron sources with no obvious optical counterparts (see e.g., [37, 38] for reviews).

The brightness fluctuations of radio halos are strictly related to the intra-cluster magnetic field structure and to the spatial distribution of the emitting particles [39]. Therefore, information on the cluster magnetic fields can be derived from detailed radio halo images. Morphological similarities between radio and X-ray images has been found in a number of clusters hosting a 
radio halo e.g., [40]. This similarity is generally valid for giant and regular halos. However, more irregular and asymmetric halos have been found in the literature. In these halos, the radio emission may show significant displacement from the X-ray emission. A statistical investigation of the offset between the radio halo and the X-ray peak of the cluster emission pointed out that halos can be quite asymmetric with respect to the X-ray gas distribution, and this becomes more relevant when halos of smaller size are considered [38, 41]. A possible explanation for this behavior can be attributed to the cluster magnetic field power spectrum structure. Indeed, Vacca et al. [42] found through a magnetic field modeling that if the outer scale of the magnetic field fluctuations is close to the observing beam, the halo results smooth and rounded. Increasing the magnetic field correlation length results in a much distorted radio halo morphology and in a significant offset of the radio halo peak from the cluster center. Cluster merger events are expected to release a significant amount of energy in the intra-cluster medium. This energy is injected on large spatial scales, and then turbulent cascades may transport energy at smaller scales, a process that is expected to affect the micro-physics of the intra-cluster medium (particles transport and acceleration, heating of the intracluster medium, and magnetic field amplification) e.g., [43, 44, 45, 46, 47, 48, 49, 50, 51]. If this amount of energy plays a role in the acceleration of relativistic particles and in the magnetic field properties (topology, amplification etc), we expect a different morphology between young and old mergers, leading younger and smaller systems to a magnetic field correlation length larger than in the more extended, and dynamical older, radio halos [41]. By correlating the information of cluster magnetic fields derived from future high resolution and high sensitivity continuum observations with the dynamical state of the clusters, as seen in the optical and X-ray band, it will be possible to confirm our expectation that galaxy clusters in process of merging might be characterized for example by a magnetic field structure which extends over size larger than in dynamically relaxed clusters.

In addition to the information derived from the continuum emission of cluster radio halos, the detection of polarized emission from radio halos is the key to investigating the magnetic field power spectrum in galaxy clusters [19, 4, 42, 52, 53]. Murgia et al. [19] simulated 3D magnetic fields in galaxy clusters with a single power-law power spectrum of the magnetic field fluctuations $\left|B_{k}\right|^{2} \propto k^{-n}$. They investigated how different magnetic field power spectra affect the shape and the polarization properties of radio halos. Models with $n>3$ and $\Lambda_{\max }>100 \mathrm{kpc}$ result in magnetic fields whose energy is larger on the large spatial scales, thus giving rise to possible filamentary and polarized radio halos. Models with $n<2$, instead, having most of the magnetic field energy on small spatial scales, will give rise to halos with a more regular morphology, and very little polarization. Typically, radio halos are found to be unpolarized when observed with the current radio facilities. Nevertheless, in agreement with what expected in Murgia et al. [19], large-scale filamentary polarized structures possibly associated with the radio halo emission have been detected so far in A2255 [54, 10], MACS J0717.5+3745 [5], and A523 [55], providing strong evidence that intracluster magnetic fields can be ordered on very large scales ( $\simeq 300-500 \mathrm{kpc})$. In Fig. 2 we show the total intensity and polarization vectors of the radio halos in which a polarized signal has been detected up to now.

The undetected polarized emission in most of the radio halos might be the result of different effects. Radio halos are faint and wide sources, therefore low resolution observations are often required to properly image their emission. If the resolution is larger than the angular scale of co- 

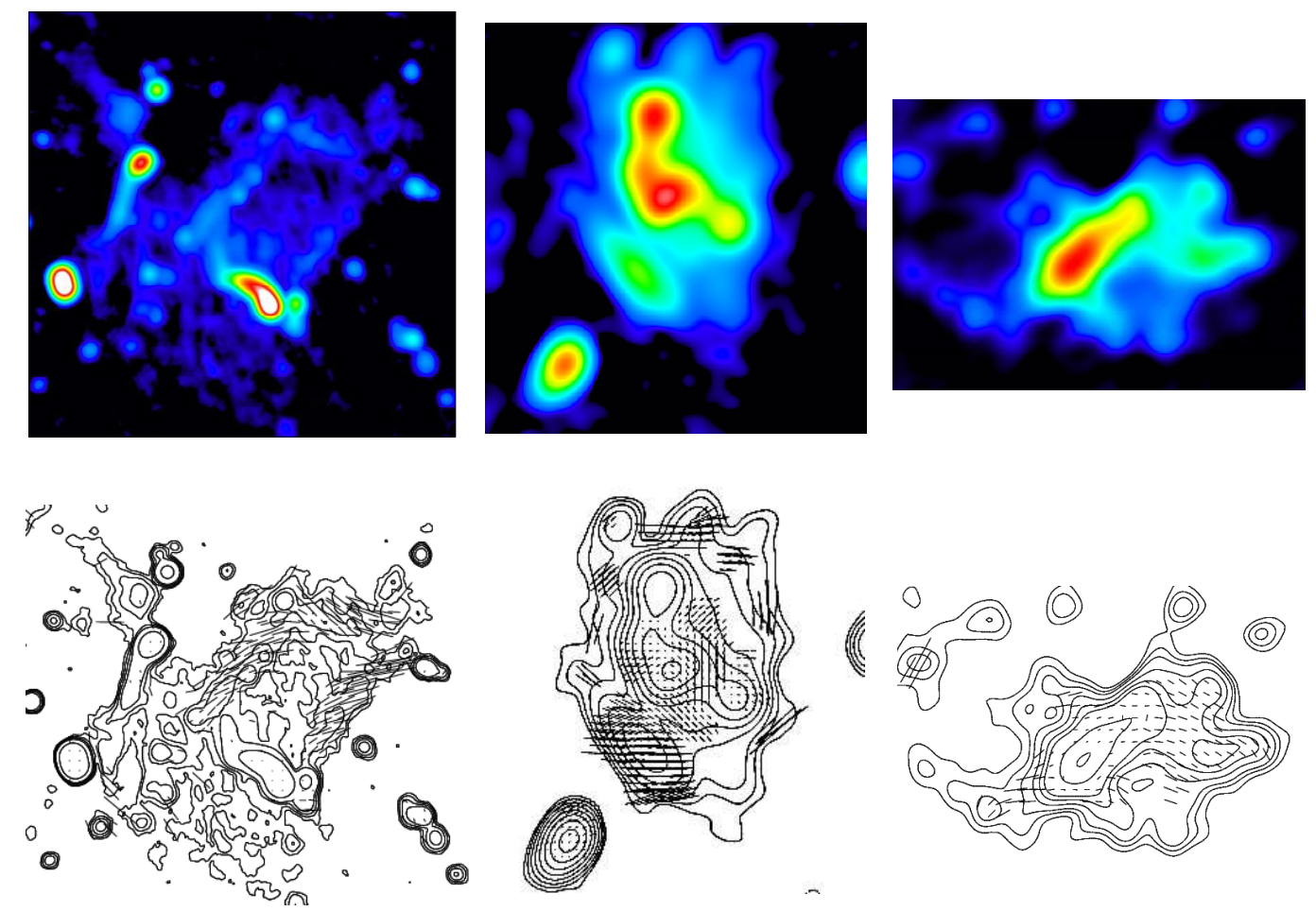

Figure 2: Total intensity (colors and contours) and polarization vectors of the radio halos in which a polarized signal has been detected up to now. Right: A2255, adapted from Govoni et al. (2005) [54]. Middle: MACS J0717.5+3745, adapted from Bonafede et al. (2009) [5]. Left: A523, adapted from Girardi et al. (2016) [55].

herent polarization patches, the so-called beam-depolarization is expected to be particularly strong. Recently, Govoni et al. [52] using cosmological magneto-hydrodynamical simulations showed that radio halos are intrinsically polarized at full-resolution. The fractional polarization at the cluster center is $\simeq 15-35 \%$ with values varying from cluster to cluster and increasing with the distance from the cluster center. However, the polarized signal is tipically undetectable if observed with the comparatively shallow sensitivity and low resolution of current radio interferometers. In addition to the effect related to the observation setup, as discussed in Sect. 3.1, the structure and the strength of the cluster magnetic field may also influence the possibility to detect polarization.

In preparation for SKA, several next-generation radio telescopes and upgrades are being constructed around the world. Among them, APERTIF, ASKAP, LOFAR, Meerkat, and JVLA all offer the chance to explore the properties of cluster diffuse emission. Deep polarization sky surveys are being planned for many of these telescopes. For example, continuum and polarization surveys like EMU (Evolutionary Map of the Universe) [56] and POSSUM (POlarization Sky Survey of the Universe's Magnetism) [57] respectively will use ASKAP to explore the northern sky at $1.4 \mathrm{GHz}$. These surveys, with a spatial resolution of $\simeq 10^{\prime \prime}$ and a sensitivity of $\simeq 10 \mu \mathrm{Jy} / \mathrm{beam}$, may play an important role in the study of non-thermal cluster physics through the analysis of total intensity and polarization of radio halos. In particular, the resolution and sensitivity values expected in future sky surveys performed at $1.4 \mathrm{GHz}$ with the SKA1 precursors and pathfinders (like ASKAP and APER- 
TIF) are very promising to detect the polarized emission of the most powerful $\left(L_{1.4 \mathrm{GHz}} \simeq 2.5 \times 10^{25}\right.$ $\mathrm{W} / \mathrm{Hz})$ radio halos, while halos of intermediate luminosity $\left(L_{1.4 \mathrm{GHz}} \simeq 2 \times 10^{24} \mathrm{~W} / \mathrm{Hz}\right)$ will be hardly detectable. In order to detect polarized signal in this kind of radio halos at a resolution of $15^{\prime \prime}$, future instruments like SKA1 should reach a polarization sensitivity of about $1 \mu \mathrm{Jy} / \mathrm{beam}$ [52].

\subsection{Numerical Simulations}

In the following we use numerical simulations to investigate how the magnetic field strength and structure can affect the polarization properties of radio halos. For this purpose, we simulated total intensity and polarization observations at $1.4 \mathrm{GHz}$ with a resolution of $15^{\prime \prime}$ of mock galaxy clusters located at a redshift $\mathrm{z}=0.08$. We modeled the gas density of the cluster with typical $\beta$ model parameters $\left(\mathrm{n}_{0}=4 \times 10^{-3} \mathrm{~cm}^{-3}, r_{\mathrm{c}}=350 \mathrm{kpc}, \beta=0.7\right)$ and, following Murgia et al. (2004), we simulated 3D magnetic fields with a single power-law power spectrum of the magnetic field fluctuations $\left|B_{k}\right|^{2} \propto k^{-n}$ and with a radial decrease of the magnetic field strength which follow the gas density $\mathrm{B}(\mathrm{r}) \propto \mathrm{n}_{e}(\mathrm{r})^{\eta}$. This magnetic field model has 5 free parameters: the central magnetic field strength $B_{0}$, the minimum and the maximum scale of the magnetic field fluctuations $\Lambda_{\min }$ and $\Lambda_{\max }$, the power law index of the power spectrum $n$, and the radial decrease of the magnetic field strength $\eta$. In the following simulations we fixed the power law index of the power spectrum to a Kolmogorov index $n=11 / 3$, the minimum fluctuations of the magnetic field to $\Lambda_{\min }=8 \mathrm{kpc}$, and the radial decrease $\eta=0.5$ and we produced a set of simulations by varying $B_{0}$ and $\Lambda_{\max }$ to investigate how these parameters can affect the total intensity and the polarized emission of radio halos. In particular, we generated synthetic radio halo images by illuminating 3D magnetic field models with a population of relativistic electrons. At each point, of the computational grid we calculated the total intensity and the intrinsic linear polarization emissivity at $1.4 \mathrm{GHz}$, by convolving the emission spectrum of a single relativistic electron with the particle energy distribution of an isotropic population of relativistic electrons. The polarization images have been obtained by taking into account that the polarization plane of the radio signal is subject to the Faraday rotation as it traverses the magnetized intracluster medium.

In Fig. 3 we fixed $\Lambda_{\max }=300 \mathrm{kpc}$ and we varied the central magnetic field strength $\left(B_{0}=0.5-\right.$ $1-5 \mu \mathrm{G})$ and the normalization of the population of the relativistic particles to obtain three halos with the same surface brightness. The images in the top show the total intensity of the radio halo emission at $1.4 \mathrm{GHz}$, while the vectors represent the orientation of the projected E-field and are proportional in length to the fractional polarization. The plot in the bottom shows the total intensity (black lines) and polarization brightness profiles ( $B_{0}=0.5 \mu \mathrm{G}$ green; $B_{0}=1 \mu \mathrm{G}$ red; $B_{0}=5 \mu \mathrm{G}$ blue). As shown in Fig. 3, given the degeneracy between magnetic fields and relativistic electrons in the synchrotron emission, it is possible to generate radio halos with identical total intensity by varying the cluster magnetic field strength. However, the degeneracy between magnetic fields and relativistic electrons is broken when polarization information are considered. Polarization is indeed strongly affected by the magnetic field strength and the theoretical fractional polarization profiles (FPOL) increase by decreasing the cluster magnetic field. This is due to the depolarization produced by the Faraday rotation effect which is stronger in higher magnetic fields. Therefore, we can conclude that future deep polarization observations have an higher chance to detect a polarized signal in clusters with lower magnetic field strength. 
In a similar way, in Fig. 4 we produced a set of simulations in which we fixed the central magnetic field $B_{0}=1 \mu \mathrm{G}$ and the population of relativistic electrons, but we varied the maximum scale of the magnetic field fluctuations $\left(\Lambda_{\max }=30-300-1000 \mathrm{kpc}\right)$. In this case, although the three clusters have a similar total intensity brightness profile, their total intensity morphology is completely different, being smooth and regular when the maximum scale of the magnetic field fluctuations is small $\left(\Lambda_{\max }=30 \mathrm{kpc}\right)$ and becoming much more irregular and filamentary by increasing $\Lambda_{\max }$. The fractional polarization profile (FPOL) increases by increasing $\Lambda_{\max }$.

To conclude, the total intensity depends on both the magnetic field and the population of relativistic electrons, while the fractional polarization mostly depends on the cluster magnetic field correlation-length and strength. Therefore, if future deep surveys will detect polarization in radio halos, it will be possible to constrain the cluster magnetic field on the basis of the fractional polarization profile, and then investigate the cluster population of relativistic electrons by fitting the observed continuum surface brightness profile. Future deep polarization observations have an higher chance to detect a polarized signal in clusters in which the magnetic field fluctuates on large scales. Indeed, current cosmological magnetic field simulations by $\mathrm{Xu}$ et al. 2012. [58], show that the cluster magnetic field is expected to fluctuate on large scales. This produce radio halos showing a filamentary morphology when observed at a sufficient high resolution. The full-Stokes capability of the future high sensitivity and high resolution spectro-polarimetric surveys, like those performed with SKA1 and its precursors and pathfinders, will permit to investigate the filamentary morphology of cluster radio halos and they may give the possibility to detect polarization for a larger sample of radio halos.

\section{Conclusions}

To obtain significant progress in the understanding of large-scale magnetic fields in the Universe, a multi-disciplinary approach is required. In fact we need to combine cutting-edge observations, data handling, theory, advanced interpretation techniques, and numerical simulations. In the near future we expect a large influx of data on large-scale magnetic fields from current and upcoming observational projects that require interpretation and confrontation with models.

Our knowledge on cluster magnetic fields will be greatly enhanced by SKA1, which will permit to study the details of total intensity and polarization of diffuse synchrotron sources and the rotation measure of radio galaxies in a large number of galaxy clusters. The high-sensitivity, low instrumental polarization contribution, and large-bandwidth will allow high-accuracy investigations of the intracluster magnetic fields in the central regions of galaxy clusters, as well as in the outskirts, where the magnetic fields are weaker. In addition, SKA1 will permit to investigate cluster magnetic fields at high redshift. Studying the cosmological evolution of fields in clusters might lead to the origin of magnetism and constrain the mechanism of their amplification to the level currently measured in the nearby Universe.

\section{References}

[1] Carilli, C.L., \& Taylor, G.B., 2002, Annual Review of Astronomy and Astrophysics, 40, 319

[2] Govoni, F., \& Feretti, L., 2004, International Journal of Modern Physics D, 13, 1549 

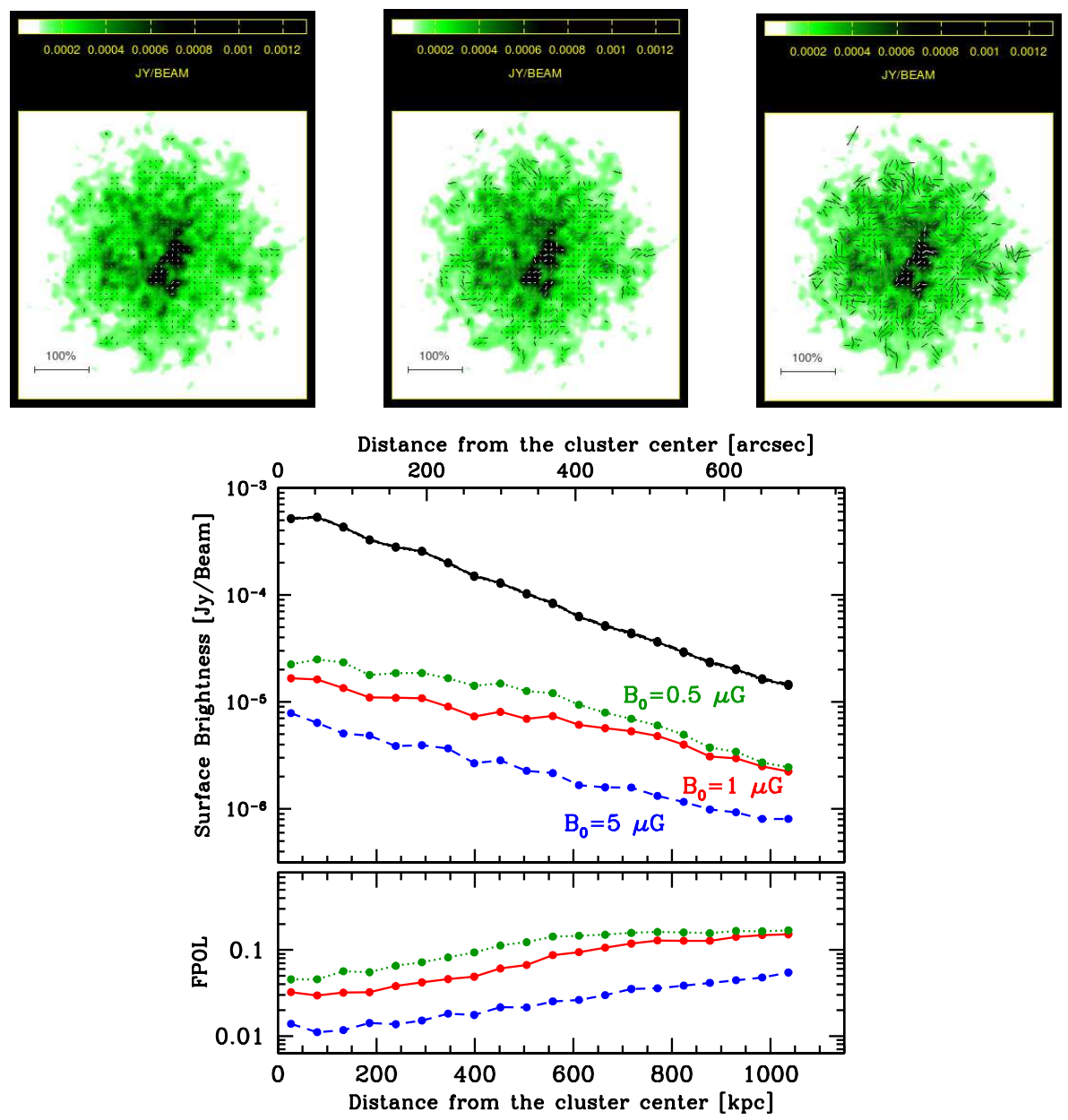

Figure 3: Top: Simulated radio halos obtained by fixing $\Lambda_{\max }=300 \mathrm{kpc}$ and by varying the central magnetic field strength $B_{0}=5 \mu \mathrm{G}$ (left), $B_{0}=1 \mu \mathrm{G}$ (middle), $B_{0}=0.5 \mu \mathrm{G}$ (right), and the normalization of the population of the relativistic particles to obtain three halos with the same surface brightness. The images (green color) indicate the total intensity of the radio halo emission at $1.4 \mathrm{GHz}$, while the vectors represent the orientation of the projected E-field and are proportional in length to the fractional polarization. Bottom: Total intensity (black lines) and polarization brightness profiles $\left(B_{0}=5 \mu \mathrm{G}\right.$ blue; $B_{0}=1 \mu \mathrm{G}$ red; $B_{0}=0.5 \mu \mathrm{G}$ green).

[3] Murgia, M., 2011, Memorie della Societa Astronomica Italiana, 82, 507

[4] Govoni, F., Murgia, M., Feretti, et al., 2006, A\&A 460, 425

[5] Bonafede, A., Feretti, L., Giovannini, G., et al. 2009, A\&A, 503, 707

[6] Burn, B.J., 1966, MNRAS, 133, 67

[7] Brentjens, M.A., de Bruyn, A.G., 2005, A\&A, 441, 1217

[8] Heald, G.H, 2009, Cosmic Magnetic Fields: From Planets, to Stars and Galaxies, Proceedings of the International Astronomical Union, IAU Symposium, Volume 259, p. 591-602

[9] Schnitzeler, D.H.F.M., Katgert, P., de Bruyn, A.G., 2009, A\&A, 494, 611

[10] Pizzo, R.F., de Bruyn, A.G., Bernardi, G., Brentjens, M.A., 2011, A\&A, 525, A104 

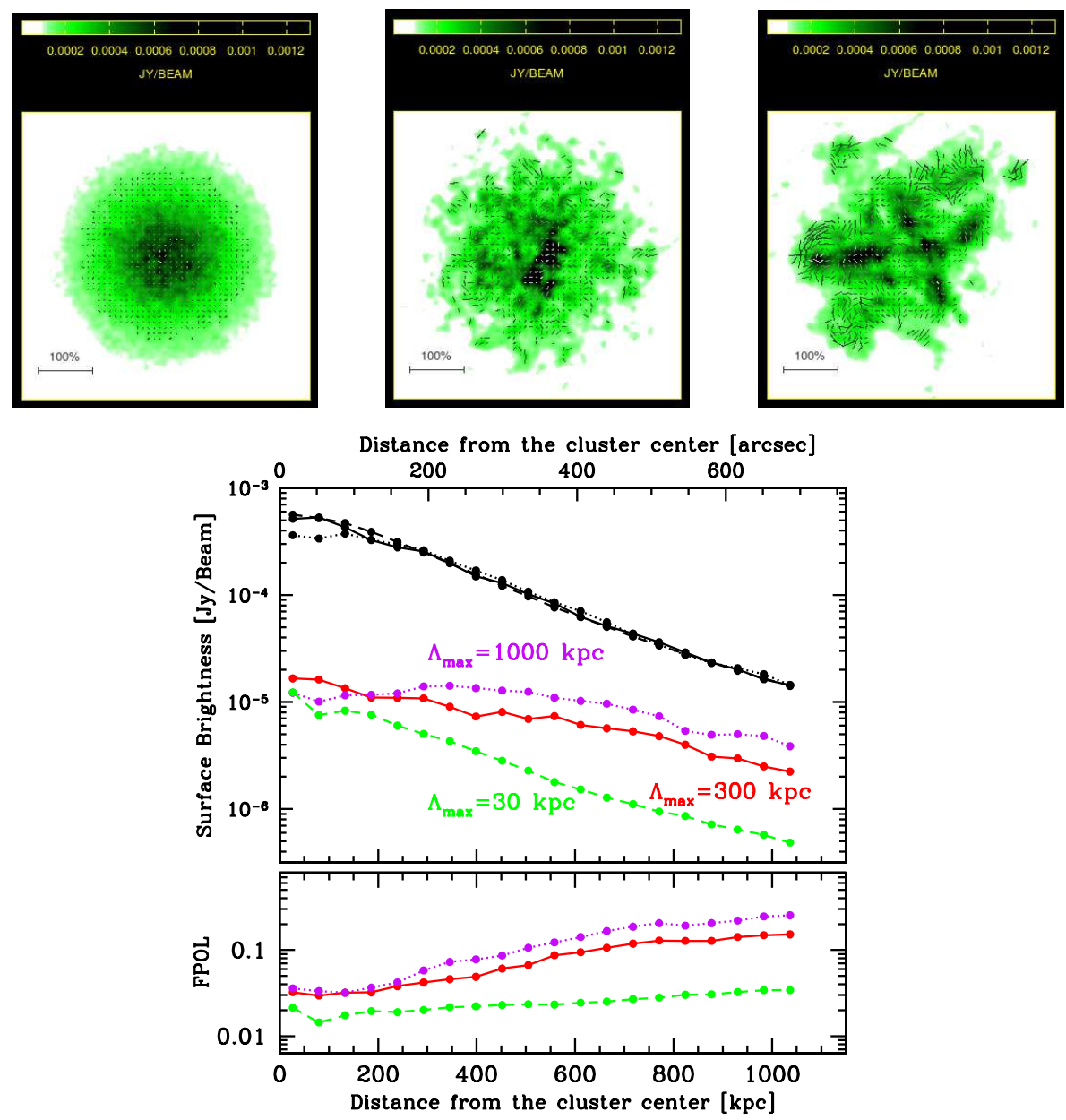

Figure 4: Top:Simulated radio halos obtained by fixing the central magnetic field strength $B_{0}=1 \mu \mathrm{G}$ and the population of relativistic electrons but by varing the maximum scale of the magnetic field fluctuations $\Lambda_{\max }=30 \mathrm{kpc}$ (left), $\Lambda_{\max }=300 \mathrm{kpc}$ (middle), $\Lambda_{\max }=1000 \mathrm{kpc}$ (right). The images (green color) indicate the total intensity of the radio halo emission at $1.4 \mathrm{GHz}$, while the vectors represent the orientation of the projected E-field and are proportional in length to the fractional polarization. Bottom: Total intensity (black lines) and polarization brightness profiles $\left(\Lambda_{\max }=30 \mathrm{kpc}\right.$ green; $\Lambda_{\max }=300 \mathrm{kpc}$ red; $\Lambda_{\max }=1000 \mathrm{kpc}$ violet).

[11] Macquart, J.-P., Ekers, R.D., Feain, I., Johnston-Hollitt, M., 2012, ApJ, 750, 139

[12] Clarke, T.E., Kronberg, P.P., Böhringer, H., 2001, ApJ 547, L111

[13] Johnston-Hollitt, M., \& Ekers, R.D., 2004, arXiv:astro-ph/0411045

[14] Govoni, F., Dolag, K., Murgia, M., et al., 2010, A\&A, 522, A105

[15] Guidetti, D., Laing, R.A., Murgia, M., et al., 2010, A\&A, 514, 50

[16] Vacca, V., Murgia, M., Govoni, F., et al., 2012, A\&A, 540, A38

[17] Bonafede, A., Feretti, L., Murgia, M., et al., 2010, A\&A, 513, 30

[18] Enßlin T.A., \& Vogt C., 2003, A\&A, 401, 835 
[19] Murgia M., Govoni F., Feretti L., et al., 2004, A\&A 424, 429

[20] Laing, R.A., Bridle, A.H., Parma, P., \& Murgia, M., 2008, MNRAS, 391, 521

[21] Kuchar, P., \& Enßlin, T.A., 2011, A\&A, 529, A13

[22] Bonafede, A., Vazza, F., Brüggen, M., et al., 2013, MNRAS, 433, 3208

[23] Vogt, C., \& Enßlin, T.A. 2003, A\&A, 412, 373

[24] Vogt, C., \& Enßlin, T.A. 2005, A\&A, 434, 67

[25] Guidetti, D., Murgia, M., Govoni, F., et al., 2008, A\&A, 483, 699

[26] Taylor, A.R., Stil, J.M., Sunstrum, C., 2009, ApJ, 702, 1230

[27] Condon J.J., Cotton W.D., Greisen E.W., et al., 1998, AJ 115, 1693

[28] Beck, R., \& Gaensler, B.M., 2004, New Astronomy Review, 48, 1289

[29] Gaensler, B.M., Beck, R., Feretti, L., 2004, New Astronomy Reviews, 48, 1003

[30] Feretti, L., \& Johnston-Hollitt, M., 2004, New Astronomy Reviews, 48, 1145

[31] Johnston-Hollitt, M., Govoni, F., Beck, R., et al., Advancing Astrophysics with the Square Kilometre Array (AASKA14), 092

[32] Rudnick, L., \& Owen, F.N., 2014, ApJ, 785, 45

[33] Stil, J.M., Keller, B.W., George, S.J., Taylor, A.R., 2014, ApJ, 787, 99

[34] Hales, C.A., Norris, R.P., Gaensler, B.M., et al., 2014, MNRAS, 441, 2555

[35] Vacca, V., Oppermann, N., Enßlin, T.A., et al. 2015, Advancing Astrophysics with the Square Kilometre Array (AASKA14), 114

[36] Vacca, V., Oppermann, N., Enßlin, T.A., et al. 2016, submitted

[37] Ferrari, C., Govoni, F., Schindler, S., et al., 2008, Space Science Reviews, 134, 93

[38] Feretti, L., Giovannini, G., Govoni, F., Murgia, M., 2012, A\&A Rev., 20, 54

[39] Tribble, P.C., 1991, MNRAS, 253, 147

[40] Govoni, F., Markevitch, M., Vikhlinin, A., et al. 2004, ApJ, 605, 695

[41] Govoni, F., Ferrari, C., Feretti, L., et al. 2012, A\&A, 545, A74

[42] Vacca, V., Murgia, M., Govoni, F., et al., 2010, A\&A, 514, A71

[43] Roettiger, K., Stone, J.M., Burns, J.O., 1999, ApJ, 518, 594

[44] Ricker, P.M., \& Sarazin, C.L., 2001, ApJ, 561, 621

[45] Dolag, K., Vazza, F., Brunetti, G., \& Tormen, G., 2005, MNRAS, 364, 753

[46] Vazza, F., Brunetti, G., Kritsuk, A., et al., 2009, A\&A, 504, 33

[47] Vazza, F., Brunetti, G., Gheller, C., Brunino, R., Brüggen, M., 2011, A\&A, 529, A17

[48] Xu, H., Li, H., Collins, D.C., Li, S., Norman, M.L., 2010, ApJ, 725, 2152

[49] Xu, H., Li, H., Collins, D.C., Li, S., Norman, M.L., 2011, ApJ, 739, 77

[50] Donnert, J., Dolag, K., Brunetti, G., Cassano, R., 2013, MNRAS, 429, 3564 
[51] Brunetti, G., \& Jones, T.W., 2014, International Journal of Modern Physics D, 23, 30007

[52] Govoni, F., Murgia, M., Xu, H., et al., 2013, A\&A, 554, A102

[53] Govoni, F., Murgia, M., Xu, H., et al., 2015, Advancing Astrophysics with the Square Kilometre Array (AASKA14), 105

[54] Govoni, F., Murgia, M., Feretti, L., et al., 2005, A\&A, 430, L5

[55] Girardi, M., Boschin, W., Gastaldello, F., et al., 2016, MNRAS, 456, 2829

[56] Norris, R.P., Hopkins, A.M., Afonso, J., et al. 2011, PASA, 28, 215

[57] Gaensler, B. M., Landecker, T. L., Taylor, A. R., and POSSUM Collaboration, 2010, Bulletin of the American Astronomical Society, 42, p.515

[58] Xu, H., Govoni, F., Murgia, M., et al., 2012, ApJ, 759, 40 